\title{
Uma análise do discurso sobre a temática acesso aberto nos anais do EDICIC Ibérico no período de 2013 a $2017^{1}$
}

\author{
Isadora Victorino Evangelista \\ Universidad Federal de São Carlos, São Carlos, São Paulo, Brasil \\ Thiago Henrique Bragato Barros \\ Universidad Federal de Rio Grande do Sul, Porto Alegre, Rio Grande do Sul, Brasil
}

\section{ORIGINAL}

\begin{abstract}
Resumo
Objetivo. Pretendeu-se analisar o conceito de acesso aberto e observar se há alguma relação com o conceito de acesso à informação enquanto um supravalor ético; como objetivos específicos, a) buscou-se investigar a presença dos termos "acesso aberto" e "dados abertos" nos eventos do EDICIC e sua relevância temática para o evento e ainda, b) divulgar os conceitos analisados e ressaltar sua importância para o avanço da ciência.

Método. Utilizou-se como fonte de pesquisa os anais do EDICIC Ibérico disponíveis em acesso aberto, ou seja, aqueles ocorridos no período de 2013-2017. Os termos de busca aplicados foram "acesso aberto" e "dados abertos", além de seus respectivos em espanhol e inglês, presentes no título, resumo e palavras-chave. O corpus gerou um total de 26 artigos, que foi posteriormente analisado, tendo como método a Análise do Discurso.

Resultados. Como resultados, foi possível chegar a um grupo de 09 instâncias éticas, identificadas a partir do discurso dos autores quando versam sobre os conceitos de Ciência Aberta e sua importância para o desenvolvimento científico.

Conclusões. $\mathrm{O}$ acesso aberto trouxe significativas mudanças na divulgação de investigações científicas, dados governamentais e pesquisas acadêmicas. No entanto, pode-se perceber que o principal fator que rege todo o movimento é o acesso aberto de maneira livre, igualitária e garantindo direitos constitucionais, evocando assim o aspecto ético que essa premissa envolve.
\end{abstract}

Palavras-chave:

Acesso aberto; Acesso à informação; Análise do Discurso; Desenvolvimento científico; Valores éticos

\section{An analysis of the discourse on the theme of open access in the annals of the lberian EDICIC in the period from 2013 to 2017}

\begin{abstract}
Objective. It was intended to analyze the concept of open access and to observe if there is any relation with the concept of access to information as an ethical supravalue; as specific objectives, a) it was sought to investigate the presence of the terms "open access" and "open data" in the events of the EDICIC and its thematic relevance for the event, and b) to divulge the analyzed concepts and highlight their importance for the scientific development.

Method. It was used as research source the records of the Iberian EDICIC available in open access, that is, those occurring in the period 2013-2017. The search terms applied were "open access" and "open data", in addition to their respective ones in Spanish and Portuguese, present in the title, abstract and keywords. The corpus generated a total of 26 articles, which was later analyzed, using Discourse Analysis as a method.

Results. As results, it was possible to reach a group of 09 ethical instances, identified from the authors' discourse when they talk about the concepts of Open Science and its importance for scientific development.

Conclusions. Open access has brought significant changes in the dissemination of scientific research, government data and academic research. However, one can see that the main factor governing the whole movement is open access in a free, egalitarian way and guaranteeing constitutional rights, thus evoking the ethical aspect that this premise involves.
\end{abstract}

Keywords:

Electronic Government; Open Data; Open Science; Public Institutions of Higher Education.

${ }^{1}$ Trabalho originalmente aceito para apresentação no IX Encontro Ibérico EDICIC 2019. 


\section{Introdução}

Tem sido discutido no âmbito da prática científica a questão da Ciência Aberta visando o desenvolvimento científico e o acesso democrático a pesquisa científica nos últimos anos com o avanço tecnológico. Esse tipo de prática é responsável por romper barreiras como a divulgação de dados de pesquisa que antes eram difundidos apenas em ambientes restritos, acesso à literatura antes garantida apenas por meio de assinaturas onerosas, alcançando ainda, a responsabilidade social pela disseminação e veracidade dessas informações compartilhadas. É possível perceber uma correlação entre o acesso aberto à ciência e o avanço do acesso à informação enquanto um supravalor ético, identificado em trabalhos como Froehlich (1994), Froehlich e Rubin (1996), Guimarães et al. (2008), Guimarães, Milani e Evangelista (2015) e Silva, Guimarães e Tognoli (2017). Embora hajam pesquisas nesse sentido, percebe-se que ainda é um número escasso, o que demonstra a necessidade de um maior desenvolvimento dessas investigações.

Como problema de pesquisa, questiona-se como dois conceitos - acesso aberto e acesso à informação enquanto valor ético - que visam o desenvolvimento científico e a responsabilidade social, podem ser vistos de maneira conjunta para alcançar melhores resultados na disseminação do conhecimento igualitário e de qualidade.

Como objetivo geral, pretendeu-se analisar o conceito de acesso aberto e observar se há alguma relação com o conceito de acesso à informação enquanto um supravalor ético; como objetivos específicos, a) buscou-se investigar a presença dos termos "acesso aberto" e "dados abertos" nos eventos do EDICIC e sua relevância temática para o evento e ainda, b) divulgar os conceitos analisados e ressaltar sua importância para o avanço da ciência.

Em um primeiro momento, buscou-se elucidar os principais pressupostos teóricos utilizados para uma melhor compreensão dos conceitos de ciência aberta, acesso aberto e dados abertos. A seguir, desenvolveu-se uma seção sobre os valores éticos em organização do conhecimento e a identificação do acesso à informação enquanto um supravalor, ou seja, um valor ético considerado superior e de extrema relevância.

Posteriormente, desenvolveu-se sobre análise do discurso, destacando suas principais correntes teóricas e significativas formas de utilização, para então, detalhar-se os métodos e procedimentos metodológicos utilizados para se chegar ao corpus subsequentemente analisado. Encerra-se então, com as principais considerações que o presente trabalho verificou.

\section{Revisão de literatura}

Embora as discussões sobre a Ciência Aberta tenham sido intensificadas com o avanço das novas tecnologias, elas não são necessariamente recentes. Costa e Leite (2017) demonstraram que seu início se dá por meio da criação do World Data Centre, na década de 50 e que possuía como principal finalidade a divulgação e compartilhamento de dados de pesquisa.

Após essa primeira iniciativa, houve outras, como na década de 70, em que a UNESCO publicou um relatório que propunha um sistema de informação científica mundial; em 1990, há o surgimento do projeto Genoma, com a proposta do armazenamento de todo dado científico relativo ao ácido desoxirribonucleico (DNA); também na década de 90, há o surgimento do programa norte-americano U.S. global Change Research Program, que prezava pelo compartilhamento dos dados de pesquisa para o efetivo desenvolvimento de pesquisas futuras; nos anos 2000 , há o surgimento de iniciativas de compartilhamento de dados, como a do National Institutes of Health e de documentos como o Declaration on Access to Research Data from Public Funding, assinada por 34 países e que expõem princípios a serem cumpridos, além disso, houve a criação do grupo Work Grouping Open Data (WGOP), que visava o acesso não apenas aberto, mas também gratuito (COSTA; LEITE, 2017).

A partir de 2011, o surgimento de políticas de acesso aberto se expande ainda mais, com a criação de fundamentos como os princípios e licenças declaradas pelo Panton Principles; a criação de grupos como o Publishing Open Data, em que editores compartilhavam os dados de pesquisas publicadas em seus periódicos; os princípios descritos pela Research Councils United Kingdom Common Principles on Data Policy, que previa um plano de divulgação dos dados em conjunto com o projeto de pesquisa a ser desenvolvida; o programa Horizon 2020, que incentivava além do compartilhamento dos dados científicos, a reutilização em diferentes pesquisas desses dados; e, em 2016, há o surgimento do acordo Statement on Data Sharing in Public Health Emergencies, que prevê a divulgação de dados sobre a superação de epidemias de saúde (COSTA; LEITE, 2017). 
Destaca-se também, o documento lançado no ano de 2018 denominado "cOAllition S", um consórcio entre treze países europeus e que garante que todas as pesquisas financiadas por iniciativas públicas deverão ser disponibilizadas em acesso aberto até 2020. O consórcio é baseado em dez princípios, identificados como "Plano S", que visam o acesso à informação científica de maneira rápida e com o mínimo de restrição (SCIENCE EUROPE, 2018). Com a intensificação de políticas a documentos nesse sentido, o conceito de Ciência Aberta demonstra ser bastante atual, ainda mais quando considerado o contexto de estruturas tecnológicas que podem auxiliar no fazer científico. Sua principal proposta se caracteriza pelo uso e disponibilização de dados de pesquisa, de maneira a serem utilizados por pesquisas futuras e como um elemento comprobatório do desenvolvimento dessas pesquisas (OLIVEIRA; SILVA, 2016).

Essa nova forma de realizar pesquisa pode ainda ser denominada como e-Science e tem como principal premissa "(...) a colaboração, compartilhamento e gerenciamento dos dados de pesquisa como aspectos principais de uma ciência colaborativa” (OLIVEIRA; SILVA, 2016, p. 05). Essa perspectiva encontra-se em evidência no cenário internacional, principalmente envolvendo âmbitos de contexto acadêmico, como agências de fomento, universidades e institutos de pesquisa.

Outra perspectiva que pode ser adotada pelo conceito de Ciência Aberta, é a democratização do acesso à literatura científica, em que essas comunicações passam a ser utilizadas de forma irrestrita, sem que sejam necessárias assinaturas ou permissão por meio de instituições e universidades. Além disso, ferramentas como softwares livres e licenças como a Creative Commons também são abarcadas por esse cenário (COSTA; LEITE, 2017).

Abagli, Clínio e Raychtock (2014) destacam ainda algumas iniciativas que podem ser identificadas na Ciência Aberta, buscando socializar a ciência de forma justa e igualitária, como por exemplo, a educação aberta e os recursos educacionais abertos, em que os materiais elaborados para a realização de atividades de ensino ficam à disposição por meio de softwares e licenças livres, prática que é facilmente encontrada nos sistemas de educação à distância. Outra iniciativa são os softwares livres, que consistem em ferramentas em que qualquer pessoa pode alterar e distribuir esses dispositivos, tornando o acesso mais democrático. A disponibilização de insumos e protocolos, para que o desenvolvimento científico seja socializado ainda que em diferentes países, também é destacado pelas autoras.

Ainda de acordo com as autoras, destaca-se o conceito de Ciência Cidadã, em que cientistas ainda inexperientes ou que não atuam como profissionais, desenvolvem iniciativas como crowd science, computação compartilhada, inteligência distribuída, sensoriamento voluntário, diálogo com a sociedade e pesquisa direta (ABAGLI; CLÍNIO; RAYCHTOCK, 2014).

Essa nova forma de compartilhamento de informações pode ser especialmente visualizada por meio dos repositórios de dados, que estão tornando-se cada vez mais populares no âmbito acadêmico. Para Sayão e Sales (2016), nessa nova dinâmica do uso abundante de dados no desenvolvimento de pesquisas, a necessidade do acesso a essas informações pode contribuir para reprodutibilidade de pesquisas e trazer uma nova forma de socialização da ciência, espaço que até então era ocupado principalmente pelas métricas em pesquisa.

As gestões desses dados encontram alicerce nos repositórios digitais de dados de pesquisa, que conseguem cumprir duas exigências atuais latentes: a integração da sociedade com os princípios expressos pela Ciência Aberta e a necessidade das agências de fomento comprovarem e registrarem os dados de pesquisas financiadas (SAYÃO; SALES, 2016). Como exemplo dessas novas práticas, cita-se o Plano de Gestão de Dados proposto pela Fundação de Amparo à Pesquisa do Estado de São Paulo, em que uma das condições para aprovação de financiamento de projetos seja que os dados oriundos das pesquisas se encontrem criteriosamente detalhados e organizados, para sua posterior disponibilização.

Percebe-se que a questão do acesso informacional é a principal finalidade do conceito de Ciência Aberta. Essa questão já foi identificada em pesquisas anteriores (GUIMARÃES et al. 2008; GUIMARÃES; MILANI; EVANGELISTA, 2015) como um supravalor ético da Ciência da Informação, mais especificamente da área de Organização do Conhecimento.

De acordo com Souza (2002), a questão do acesso à informação evidencia um poder que os profissionais da Ciência da Informação possuem, em que os indivíduos podem participar de maneira cooperativa ou excludente, representando da maneira correta os materiais ou negligenciando os documentos e ferramentas de organização do conhecimento. 
Segundo White (1991), a questão do acesso à informação pode ser caracterizada com o maior problema ético identificado em unidades de informação e pode ser definida como uma responsabilidade social da instituição, em que a informação deve ser disponibilizada de maneira igualitária a qualquer indivíduo, independente de suas camadas sociais, níveis de instrução ou pontos de vista.

Esse conceito evoca ainda a importância de se evitar a discriminação em ambientes informacionais, fato que é destacado por Bunge (1991). Para o autor, essa ideia corresponde à diligência e zelo do profissional enquanto forma de se evitar práticas antiéticas.

Para Morrison (1991), a questão do acesso informacional encontra oposições em dois âmbitos: equidade de acesso e qualidade do serviço prestado, oferecendo informação relevante e pertinente. Para o autor, esses temas possuem uma especial dimensão ética quando em relação às minorias, pois as unidades devem conhecer suas individualidades sociais, culturais e demográficas, além de suas necessidades informacionais. Após conhecer esses aspectos, é necessário que a instituição se esforce para que haja a inclusão desse grupo, por meio de objetivos traçados e projetos específicos pra esse tipo de público.

Ainda de acordo com o autor, devem ser reforçados quatro tópicos: acesso à coleção - é preciso que barreiras culturais, educacionais e linguísticas, sejam reconhecidas para que sejam evitadas, de nada adianta uma seleção criteriosa de recursos que incluam essas minorias, se o processamento técnico desse material não é feito da maneira correta; funcionários da biblioteca - os funcionários devem ser orientados sobre como lidar com essa coletividade, além de estarem cientes das barreiras e conhecer a literatura que possa interessar; programação específica - em que os temas e os participantes reflitam a etnia da comunidade e que haja uma divulgação efetiva sobre essas programações; instalações - elas devem estar apropriadas para receber as minorias, mesmo que as demandas ainda não sejam explícitas (MORRISON, 1991).

Em relação às ferramentas de organização do conhecimento e sua relação com o acesso informacional, destacam-se as iniciativas de Bair (2005), que preocupou-se em elaborar um código de ética para os profissionais catalogadores, de forma a promover o acesso à documentos e Van der Walt (2004) que demonstra essas preocupação em linguagens de indexação, que possuem como premissa básica dar acesso e, portanto, devem considerar que diferentes usuários possuem necessidades informacionais diferentes e os termos de indexação devem refletir essa pluralidade, buscando sempre um certo nível de subjetividade e que a busca por uma teoria sedimentada para essas questões deve ser constantemente perseguida.

Em 2008, Guimarães et al. identificaram o acesso à informação como um valor que seria incluído em esfera superior, que deveria guiar todas a atividade informacional, juntamente com outros valores como direito autoral, liberdade, equidade, segurança, diversidade, minimização de riscos, todos também relacionados com o conceito de Ciência Aberta.

Em 2015, Guimarães, Milani e Evangelista confirmam essa constatação, destacando o acesso informacional como um supravalor, que se revela como um guiador para a obtenção de todos os outros valores éticos identificados na Organização do Conhecimento.

No tocante às atividades de descrição e classificação arquivísticas, Silva, Tognoli e Guimarães (2017) também identifica o acesso informacional enquanto um valor ético superior aos outros, por aparecer em uma maior ocorrência e que evidencia que as atividades de representação desenvolvidas pelo arquivista são sempre visando o acesso desses documentos pelo público usuário.

Além do acesso informacional, outra perspectiva que é alterada com a disseminação do conceito de Ciência Aberta e seu auxílio para o desenvolvimento científico e social, é a concepção de autoria. Para Oliveira e Silva (2016), sob essa nova ótica, inclusive os bibliotecários e arquivistas podem ser compreendidos como autores, visto que representam e disponibilizam os dados de pesquisa, de maneira a serem recuperados e utilizados em novas investigações.

Para as autoras, há uma diferença entre o pesquisador-autor primário, que produz os primeiros dados e as autorias derivadas e coletivas, que serão concedidas a partir do contexto - que pode ser científico, governamental, cultural ou artístico - e a responsabilidade de suas atribuições. Dessa maneira, o autor deixa de possuir unicamente os registros e dados de pesquisa, para ser um compilador, que reúne os materiais para que sejam gerados novos conhecimentos. 
A Analise do Discurso (doravante denominada $A D$ ) trata-se de uma teoria comprometida com as questões do sentido do texto em sua circunscrição social, histórica e ideológica assim, supra-valores, como aqueles que buscamos trabalhar ao longo deste trabalho, tem uma questão discursiva fundamental. AAD trata-se de uma forma de analisar e identificar enunciados, posições ideológicas, formações discursivas no interior das produções textuais. Em resumo para a análise do discurso a produção textual funciona como um objeto de inscrição do discurso e da ideologia, o discurso é então um espaço aberto, que parte da língua, já que a materialidade é o texto e é atravessado pela ideologia, circunscrito por sua própria história. Na literatura sobre Análise do Discurso é possível encontrar, atrelada ao conceito de discurso, a metáfora que relaciona o discurso com uma rede de sentidos que se transformam em um tecido discursivo. (BARROS, 2015). Assim o discurso e um sistema, mas um sistema aberto, passível de mudandas e deslocamentos.

Existindo, então "furos" no texto que rementem a sua construção social, ideológica, histórica, interpelados pela ideologia e pelo subconsciente. Por isso, pode-se dizer que o discurso é a "palavra em movimento, prática de linguagem" (ORLANDI, 2007, p.15).

Para fins deste trabalho, analisar o discurso significa reconhecer os padrões identificar as relações entre os textos e a enunciação de uma ciência aberta, democrática na qual todos tem o direito de conhecer os processos e prodecimentos científicos. Significa então, que a produção cientifica busca desvencilhar-se de amarras corporativas e fechadas.

Buscando assima de tudo em uma posição discursivo ideológica abrir o processo de divulgação cientifica a comunidade que produz, legitima e constrói a ciência. Apremissa básica passa a ser então o acesso, para além da questão única e exclusiva da divulgação cientifica.

Ao longo do texto, essa questão fundamental da enunciação, ou seja, da formação discursiva, que é uma posição, uma convergência de enciados a uma perpectiva aberta e demoratica, busca ficar evidente nos textos que analisa.

\section{Metodologia}

A presente pesquisa caracteriza-se como bibliográfica, por utilizar como objeto de pesquisa artigos publicados nos anais dos eventos EDICIC Ibérico. Ela também possui caráter quantitativo, por utilizar-se de alguns dados estatísticos como representação de resultados e qualitativo, por utilizar-se da Análise do Discurso pra identificar relações implícitas, ainda que não declaradas de maneira evidente, entre o conceito de acesso aberto e acesso informacional, como um supravalor ético. A análise discursiva toma por base a metodologia desenvolvida por Barros (2017), em relação aos enunciados elencados.

Como citado, a fonte de pesquisa foram os anais publicados resultantes dos eventos EDICIC Ibérico. O evento é realizado bianualmente desde 2008, no entanto, considerando que os eventos de 2008 a 2011 foram publicados em formato impresso, utilizou-se como objeto de pesquisa os anais dos eventos realizados em 2013, em Porto; de 2015, ocorrido em Madrid e o último evento, em 2017, sucedido em Coimbra. A escolha desse material deve-se ao fato desses documentos serem disponibilizados de maneira aberta e online, facilitando assim, a busca dos artigos.

Como termos de pesquisa, foram utilizados "acesso aberto", "dados abertos", além de seus respectivos termos em inglês - open access e open data - e em espanhol - acceso abierto e datos abiertos. Dada a natureza internacional do evento, fez-se necessário a escolha da busca de termos nesses três idiomas.

Esses termos foram pesquisados nas áreas de maior conteúdo informacional do documento, a saber: títulos, resumos e palavras-chave. Os autores reconhecem a presença de artigos que trabalham com a temática no desenvolvimento do artigo ou ainda, utilizam-se de termos similares aos definidos pela pesquisa, no entanto, por uma questão de rigor metodológico, optou-se por selecionar para o corpus apenas os artigos que continham os termos selecionados nas áreas definidas.

O corpus gerou um total de 26 artigos, que podem ser verificados no quadro a seguir: 
Quadro 1 - Corpus de pesquisa

\begin{tabular}{|c|c|c|}
\hline Dados dos artigos & País & Termo utilizado \\
\hline $\begin{array}{l}\text { SÁ; M. I. F.; SOUZA, R. F. Bibliotecas } \\
\text { digitais: visibilidade e organização global, } \\
2013 .\end{array}$ & Brasil & Acesso aberto \\
\hline $\begin{array}{l}\text { RODRIGUES, C. T.; RASSA, M. A. L. Uso } \\
\text { de las herramientas de la web social por } \\
\text { los investigadores de la Universidad de } \\
\text { Salamanca: estúdio preliminar, } 2013 .\end{array}$ & Espanha & Acesso aberto \\
\hline $\begin{array}{l}\text { AGUIRRE-LIGUERA, N.; CERETTA, M. } \\
\text { G. Construyendo um modelo de repositó- } \\
\text { rio de acceso abierto para Uruguay, } 2013 .\end{array}$ & Uruguai & Acesso aberto \\
\hline $\begin{array}{l}\text { TARDENCILLA-MARENCO, E. M.; } \\
\text { GUANBE; M. F.; ALVES, F. M. M. Acceso } \\
\text { abierto em África: dificultades y desafios, } \\
2015 .\end{array}$ & Nicarágua e Espanha & Acesso aberto \\
\hline $\begin{array}{l}\text { MEDEIROS, F.; GONZÁLEZ, J. A. M. } \\
\text { Profissionais da informação e medieva- } \\
\text { listas em torno da construção de uma } \\
\text { taxonomia de história medieval portugue- } \\
\text { sa: dificuldade e reptos, } 2015 \text {. }\end{array}$ & Portugal & Acesso aberto \\
\hline $\begin{array}{l}\text { LEITÃO, H.; BORGES, M. M.; SIMÕES, } \\
\text { G. M. Resumos científicos em saúde: es- } \\
\text { tudo exploratório de resumos de artigos } \\
\text { da Nature PLoS One, } 2015 \text {. }\end{array}$ & Portugal & Acesso aberto \\
\hline $\begin{array}{l}\text { COBO-SERRANO, S.; RAMOS-SIMÓN, } \\
\text { L. F.; ARQUERO-AVILÉS, R. La normati- } \\
\text { va RISP em los websites de la Adminis- } \\
\text { tración General del Estado, } 2015 .\end{array}$ & Espanha & Datos abiertos \\
\hline $\begin{array}{l}\text { MARTÍN-GONZÁLEZ; Y.; SAINZ-IGLE- } \\
\text { SIAS, P. Open University data em Espa- } \\
\text { ña: de los portales de transparência a los } \\
\text { catálogos de datos abiertos, } 2015 .\end{array}$ & Espanha & Datos abiertos \\
\hline $\begin{array}{l}\text { GUERRA-GONZÁLEZ, J. T. Recursos } \\
\text { ditales docentes em acceso abierto em el } \\
\text { portal, } 2015 .\end{array}$ & México & Acceso abierto \\
\hline $\begin{array}{l}\text { MARCONDES, C. H. O papel dos mode- } \\
\text { los conceituais para interoperabilidade } \\
\text { entre acervos digitais de arquivos, biblio- } \\
\text { tecas e museus, } 2015 \text {. }\end{array}$ & Brasil & Dados abertos \\
\hline $\begin{array}{l}\text { CAMPOS, L. M.; BARBOSA, N. T. Apli- } \\
\text { cação de dados abertos ligados basea- } \\
\text { dos em ontologias, } 2015\end{array}$ & Brasil & Dados abertos \\
\hline $\begin{array}{l}\text { QUARESMA, H.; BORGES, M. M. } \\
\text { Inclusão digital: a acessibilidade das pá- } \\
\text { ginas web dos repositórios institucionais } \\
\text { para deficientes, } 2015 .\end{array}$ & Portugal & Acesso aberto \\
\hline $\begin{array}{l}\text { COSTA; T.; ALVIM, L. Uma análise bi- } \\
\text { bliométrica das publicações sobre acesso } \\
\text { aberto indexadas na Web of Science, } \\
2017\end{array}$ & Portugal & Acesso aberto \\
\hline $\begin{array}{l}\text { FREITAS, M. C. V. Revistas especializa- } \\
\text { das em termas arquivísticos de acesso } \\
\text { aberto, } 2017 .\end{array}$ & Portugal & Acesso aberto \\
\hline $\begin{array}{l}\text { RIOS; F. P.; LUCAS; E. R. O.; AMORIM, } \\
\text { Igor Soares. Manifestos do movimento de } \\
\text { acesso aberto: uma análise de domínio, } \\
2017 .\end{array}$ & Brasil & Acesso aberto \\
\hline
\end{tabular}




\begin{tabular}{|c|c|c|}
\hline $\begin{array}{l}\text { ANTUNES, M. L. et al. Literacia da infor- } \\
\text { mação: o primeiro degrau para a ciência } \\
\text { aberta, } 2017 \text {. }\end{array}$ & Portugal & $\begin{array}{c}\text { Acesso aberto e dados } \\
\text { abertos }\end{array}$ \\
\hline $\begin{array}{l}\text { ALVES, F. M. M.; ALVES, R. M. Curadoria } \\
\text { digital: situação e perspectivas no Brasil, } \\
\text { 2017. }\end{array}$ & Brasil & Acesso aberto \\
\hline $\begin{array}{l}\text { LEITÃO, H.; BORGES, M. M. Open ac- } \\
\text { cess e serviço de referência - do excesso } \\
\text { à essência. In: BORGES, Maria Manuel; } \\
\text { SANZ-CASADO, Elias (Coord.), } 2017 .\end{array}$ & Portugal & Open access \\
\hline $\begin{array}{l}\text { CIRINO, S. D. et al. Política pública de } \\
\text { periódicos científicos para o estado de } \\
\text { Minas Gerais (Brasil), } 2017 .\end{array}$ & Brasil & Acesso aberto \\
\hline $\begin{array}{l}\text { OLIVEIRA, C.; PASTORINI, V. Portais de } \\
\text { periódicos científicos: aspectos de visibili- } \\
\text { dade e institucionadade, } 2017 .\end{array}$ & Brasil & Acesso aberto \\
\hline $\begin{array}{l}\text { PINHEIRO, L.V. Políticas públicas, ações } \\
\text { e manifestos de ciência aberta para pes- } \\
\text { quisa brasileira, } 2017 .\end{array}$ & Brasil & Acesso aberto \\
\hline $\begin{array}{l}\text { MELO, L. B. et al. Os espaços } \\
\text { das bibliotecas académicas como camin- } \\
\text { ho para o conhecimento: um estudo de } \\
\text { caso na Universidade de Lisboa, } 2017 .\end{array}$ & Portugal & Acesso aberto \\
\hline $\begin{array}{l}\text { MACEDO, L. S. Ascensão. O caso dos } \\
\text { fundos conventuais madeirenses dis- } \\
\text { persos entre o arquivo nacional torre do } \\
\text { tombo e o arquivo regional e biblioteca } \\
\text { pública da Madeira, } 2017 .\end{array}$ & Portugal & Acesso aberto \\
\hline $\begin{array}{l}\text { MACIEL; R. S.; ZAFALON, Z. R. Critérios } \\
\text { para a escolha de padrões de metadados } \\
\text { e de softwares para repositórios institu- } \\
\text { cionais, } 2017 \text {. }\end{array}$ & Brasil & Acesso aberto \\
\hline $\begin{array}{l}\text { FILIPPO, D. ; SILVA, P.; BORGES, M. M. } \\
\text { Caracterización y presencia en las redes } \\
\text { sociales de las publicaciones científicas } \\
\text { sobre open service, } 2017 .\end{array}$ & Espanha e Portugal & Open data \\
\hline $\begin{array}{l}\text { PALETTA, F. C.; SILVA, A. M. Digital } \\
\text { library and the information technology } \\
\text { lifecycle, } 2017 .\end{array}$ & Brasil e Portugal & Open data \\
\hline
\end{tabular}

Fonte: elaborado pelos autores.

É possível perceber que a temática acesso aberto tem tido um constante crescimento nos eventos Edicic lbérico, especialmente no último evento, que é responsável por mais da metade dos artigos selecionados para o corpus. Embora no evento de 2015 já apareça o acesso aberto como um dos temas do encontro, em 2017 o título do evento é especificamente sobre a contribuição da Ciência Aberta para a sociedade.

Em relação aos idiomas dos trabalhos, houve 20 trabalhos em português, 7 trabalhos em espanhol e 1 único trabalho em inglês, porém, escrito por autores brasileiros. Essa forte incidência de trabalhos em português, deve-se ao fato de o Edicic Ibérico ser um forte canal de comunicação para portugueses e brasileiros, com uma intensa participação desses países.

Verifica-se que o termo mais utilizado pelos autores é justamente o termo mais geral - "acesso aberto" em português, fato que também se justifica pela maioria dos artigos do corpus serem nesse idioma. Embora tenha sido recuperado apenas um artigo em inglês, constata-se que os termos nessa língua são utilizados em artigos de outros idiomas, por se tratar do termo no original. 


\section{Resultados}

Após a realização da análise nos documentos selecionados, por meio de metodologia proposta por Barros (2017), foi possível perceber que o acesso informacional pode ser considerado um supravalor ético que rege toda a filosofia encontrada nos movimentos de Ciência Aberta e, para comprovar esse fator, algumas formações discursivas podem ser destacadas, conforme verificado nos quadros a seguir:

\section{Quadro 01 - Manifestação contra preços abusivos}

\section{Enunciações/superfície linguística}

"El movimiento de AA surge por la confluencia de varios elementos, entre los que se destacan: la oportunidad que brinda Internet para la difusión, publicación y acceso a contenidos y datos de investigaciones; y sobre todo la presión ejercida por las grandes empresas editoriales que exigen pagos de suscripción cada vez más elevados para acceder al conocimiento registrado en sus revistas, reteniendo para sí los derechos de copyright" (AGUIRRE-LIGUIERA; CERETTA, 2013, p. 1211)

"superação do paradigma da comunicação científica mediada pelas grandes editoras comerciais por uma comunicação de acesso livre via internet" (RIO; LUCAS; AMORIM, 2017, p. 232)

"Em paralelo, o Movimento de Acesso Aberto trouxe uma nova filosofia para a produção e comunicação científicas. Esse movimento eclodiu a partir da crise dos periódicos, na qual os preços elevados das assinaturas e os excessivos embargos para o acesso a eles vinham dificultando a comunicação das pesquisas realizadas pelas instituições acadêmicas com recursos públicos." (MACIEL; ZAFALON, 2017, p. 1357)

\section{Esquecimento $n^{\circ} 02 /$ Paráfrases}

"superação do paradigma"

"nova filosofia"

Esquecimento $n^{0} 01 /$ Discurso

O contexto do surgimento do movimento Ciência Aberta desponta, principalmente, como forma de protesto contra o preço elevado que as editoras comerciais cobravam pelas assinaturas de periódicos científicos e que prejudicava grande parte das bibliotecas públicas. Os autores compartilham da ideia que, na época dessa crise científica impulsionada pelas editoras, escrever de maneira que estivesse disponível a todos, era ainda considerado uma utopia.

Fonte: elaborado pelos autores (2019).

\section{Quadro 02 - Acesso igualitário ao conhecimento científico}

\section{Enunciações/superfície linguística}

"El fundamento teórico del proyecto se sustenta en la filosofía del movimiento de acceso abierto (AA) que propone que el conocimiento científico debe estar disponible em forma gratuita y libre a través de internet, para que todos los usuarios puedan utilizarlo con cualquier propósito legal, siempre que se garantice a sus autores la integridad de su trabajo y el derecho a ser debidamente reconocidos y citados (Budapest Open Access Initiative, 2002). De esta manera se busca promocionar la generación de nuevo conocimiento y por tanto el desarrollo social y científico de la humanidad en su conjunto, franqueando las barreras entre países pobres y ricos" (AGUIRRE-LIGUIERA; CERETTA, 2013, p. 1211)

"Enquanto na América do Norte e Europa, a necessidade de acesso aberto surge por causa dos valores exorbitantes cobrados no acesso aos artigos, na América Latina a questão é motivada pela falta de publicação científica no idioma local, além da cobrança que inviabilizava o acesso de países pobres e emergentes aos conteúdos essenciais à produção científica" (RIO; LUCAS; AMORIM, 2017, p. 228)

"Numa sociedade em que se prevê que se caminhe cada vez mais para a igualdade de oportunidades, de direitos e de deveres, esta tem no Acesso Aberto à informação e ao conhecimento, uma ponte para atingir o grande objetivo que é o crescimento do ser humano com vista à plena igualdade" (LEITÃO; BORGES; SIMÕES, 2015, p. 08)

"Dicho instrumento legal tenía como objeto la armonización del acceso a los documentos públicos en todos los organismos de los países miembros de la Unión Europea, el establecimiento de un principio de igualdad y no discriminación en el acceso y uso de la información pública o el establecimiento de un esquema transparente de precios para dicho acceso (Directiva 2003/98CE)". (COBO-SERRANO; RAMOS-SIMÓN; ARQUERO-AVILÉS, 2015, p. 02). 
"A Web e os formatos digitais de conteúdos tornam as instituições de memória e de cultura seus acervos acessíveis a um público muito mais amplo do que seria possível de forma presencial. A Rede vem se tornando um instrumento sem precedentes para a disseminação desses acervos, a um público cada vez muito mais amplo, a qualquer hora, de qualquer lugar, através de dispositivos como computadores convencionais até "smartphones" (HSI, 2002). A Web pode contribuir assim para democratizar o acesso a estes conteúdos, antes só disponíveis ao público presencial" (MARCONDES, 2015, p. 12)

"Os repositórios científicos de acesso aberto constituem valiosas oportunidades de acesso livre e gratuito ao conhecimento produzido nas instituições de ensino superior. Para cumprirem plenamente a sua missão, os repositórios não devem apresentar qualquer barreira de acesso à informação, particularmente para os utilizadores que apresentem algum tipo de deficiência, como a deficiência visual” (QUARESMA; BORGES, 2015, p. 08)

"Em meio a essas questões, encontra-se o movimento do Acesso Aberto, opção que veio incrementar a difusão das revistas, especialmente em contextos em que são parcos os recursos e os argumentos usados na sua divulgação." (FREITAS, 2017, p. 212)

"A questão dos arquivos expatriados a nível intranacional requer uma abordagem que aproximem as entidades custodiantes e a participação das comunidades reivindicantes para encontrar soluções que satisfaçam direitos fundamentais, como o acesso à informação e à memória" (MACEDO, 2017, p. 1335)

\section{Esquecimento $n^{\circ} 02 /$ Paráfrases}

"crescimento do ser humano com vista à plena igualdade"

"principio de igualdad y no discriminación en el acceso y uso de la información pública"

"incrementar a difusão"

"direitos fundamentais"

\section{Esquecimento $n^{0} 01 /$ Discurso}

A partir do quadro anterior, foi possível identificar esta segunda instância, que demonstra que não apenas o acesso à informação deveria ser o foco do movimento acesso aberto, mas também o acesso por todos de maneira igual, equiparando o acesso de países desenvolvidos e subdesenvolvidos a publicações científicas, criando o que foi denominado "bem social". Essa questão se sobressai nos países latinos, porque aumenta a possibilidade de publicações no idioma desses países, quebrando uma barreira de predomínio da língua inglesa. Esse acesso igualitário é declarado por alguns autores como um direito constitucional, evitando a discriminação científica; democratizando o acesso e evocando uma ideia de poder propiciado pelo acesso informacional.

Fonte: elaborado pelos autores (2019).

Quadro 03 - Retorno dos financiamentos governamentais

Enunciações/superfície linguística

"Este proyecto no tiene antecedentes a nivel nacional y aspira a contribuir a la toma de decisiones políticas sobre Ciencia y Tecnología (СуT) que favorezcan la democratización del conocimiento social, científico y tecnológico generado en el país. Se focaliza particularmente en la investigación financiada con fondos públicos, que en el país representa una alta proporción de las actividades investigativas." (AGUIRRE-LIGUIERA; CERETTA, 2013, p. 1210)

"Las iniciativas incluidas en el movimiento Open Science tienen como objetivo permitir la distribución, utilización y reutilización de los datos generados por los investigadores durante el proceso de investigación que han llevado a cabo y que a menudo, está sufragado con fondos públicos" (MARTíN-GONZÁLEZ, SAINZ-IGLESIAS, 2015, p. 06)

"En concreto, las políticas de transparencia responden a la necesidad de rendir cuentas ante la ciudadanía para lo que se requiere, por un lado, el cumplimiento del Principio de publicidad activa y, por otro, la regulación del derecho de acceso a la información de las instituciones sufragadas con fondos públicos entre las que se encuentran las universidades" (MARTÍN-GONZÁLEZ, SAINZ-IGLESIAS, 2015, p. 06)

"Dicho instrumento legal tenía como objeto la armonización del acceso a los documentos públicos en todos los organismos de los países miembros de la Unión Europea, el establecimiento de un principio de igualdad y no discriminación en el acceso y uso de la información pública o el establecimiento de un esquema transparente de precios para dicho acceso (Directiva 2003/98CE)". (COBO-SERRANO; RAMOS-SIMÓN; ARQUERO-AVILÉS, 2015, p. 02). 
"Acredita-se que um sistema de publicação de pesquisa de AA estável, competitivo e com qualidade assegurará benefícios imensos, não só para estudos, mas também para a sociedade como um todo. A publicação académica é uma atividade global, e as organizações que realizam e apoiam a pesquisa, particularmente as que usam dinheiro público, têm a responsabilidade nesta matéria." (COSTA; ALVIM, 2017, p. 115)

Esquecimento $n^{\circ} 02 /$ Paráfrases

"sufragado com fondos públicos"

"rendir cuentas ante la ciudadanía"

"regulación del derecho de acceso a la información de las instituciones sufragadas con fondos públicos" "esquema transparente de precios para dicho acceso"

"particularmente as que usam dinheiro público"

\section{Esquecimento $n^{0} 01 /$ Discurso}

Outra instância que pode ser caracterizada é o fato de que, permitir o acesso aberto às pesquisas científicas é uma obrigação moral, visto que geralmente essas pesquisas são financiadas pelo governo que por sua vez, recebe seus recursos por meio de impostos pagos pela sociedade. Ademais, a disponibilização desses dados gera uma economia governamental, tanto financeiramente quanto em relação ao tempo gasto para realização de pesquisas.

Fonte: elaborado pelos autores (2019).

Quadro 04 - Memória institucional

Enunciações/superfície linguística

"Os repositórios digitais (RDs) são bases de dados online que reúnem, de maneira organizada, a produção científica de uma instituição ou área temática. Os RDs armazenam arquivos de diversos formatos. Constituem-se como preciosas ferramentas de trabalho, tanto para os pesquisadores, quanto para as instituições ou sociedades científicas, proporcionando maior visibilidade aos resultados de pesquisas e possibilitando a preservação da memória científica das diversas instituições" (QUARESMA; BORGES, 2015, p. 05)

"Sin embargo, estudios actuales (KELLY; EELLS, 2015) informan sobre los esfuerzos que África realiza con el acceso abierto y como la creación de repositorios pueden vencer la brecha del conocimiento exponiendo sus investigaciones, archivando su propia literatura y ser utilizados en los procesos de enseñanza-aprendizaje" (TARDENCILLA-MARENCO; GUANBE; MELO-ALVES, 2015, p. 02)

"Sudáfrica, es el país que más está desarrollado en el uso del acceso abierto a través de la vía verde, teniendo más disponibilidad de repositorios institucionales. Sus bibliotecarios están conscientes que para seguir avanzando en una sociedad del conocimiento, las universidades deben aumentar la producción y divulgación de su investigación" (TARDENCILLA-MARENCO; GUANBE; MELO-ALVES, 2015, p. 10)

"Em consequência, a gestão da informação desenvolveu métodos e ferramentas adequadas para traduzir os dados em informações, promover seu compartilhamento, preservação e reuso, de forma livre, e promover novas práticas de gestão de dados digitais." (ALVES; ALVES, 2017, p. 71)

"No Brasil, o Instituto Brasileiro de Informação em Ciência e Tecnologia (IBICT) foi pioneiro na área da preservação digital, ao aderir, em 2013, ao Programa Lots of Copies Keep Stuff Safe (LOCKSS) da Stanford University, que permitiu a preservação da informação científica brasileira e o acesso a publicações de grandes instituições internacionais, participantes do referido programa." (ALVES; ALVES, 2017, p. 73)

Esquecimento $n^{0} 02 /$ Paráfrases

"possibilitando a preservação da memória científica das diversas instituições"

"preservação e reuso"

"preservação da informação científica brasileira"

\section{Esquecimento $n^{\circ} 01 /$ Discurso}

Os repositórios institucionais e as bibliotecas digitais, enquanto espaços que armazenam documentos open access podem ser refletidos enquanto produtores da memória institucional e acadêmica das entidades responsáveis por eles Além disso, esse tipo de prática torna-se relevante para a preservação de registros.

Fonte: elaborado pelos autores (2019). 
Quadro 05 - Mudança paradigmática em unidadles de informação

\section{Enunciações/superfície linguística}

"Relações como as citadas potencializam-se na medida que objetos destes acervos passam a ser representados como objetos digitais e disponibilizados na Web. A Web traz o potencial de integrá-los todos e integrá-los ainda com lugares, pessoas, eventos, épocas, estilos, movimentos artísticos, culturais, políticos e sociais." (MARCONDES, 2015, p. 09)

"África sigue uniendo esfuerzos entre sus países, para dar avances más significativos en el uso del acceso abierto. Entre la comunidad académica y en las universidades africanas, se tienen que superar obstáculos culturales para la integración de repositorios en su gestión académica y para reconocer el potencial y valor de estos para la investigación. El uso del acceso abierto a través de la vía verde es una oportunidad para las instituciones de educación en el continente negro para que logren tener una mayor disponibilidad y rápida repercusión del conocimiento que se genera." (TARDENCILLA-MARENCO; GUANBE; MELO-ALVES, 2015, p. 03)

"Também em 2003 foi constituída a Declaração de Berlim que traz como novidade o foco nas Humanidades como área que também necessita de direcionamento para o caminho do Movimento. O documento destaca, além de bibliotecas e arquivos, os museus como importantes produtores de conhecimento e, dessa forma, com necessidade para dispor suas produções em repositórios abertos. Destaca, ainda, que a internet deve ser sustentável, interativa e transparente e que as ferramentas de softwares devem ser acessíveis e compatíveis. Coloca o acesso aberto como fonte ao conhecimento científico e do patrimônio cultural." (RIO; LUCAS; AMORIM, 2017, p. 233)

“É esta declaração que inaugura o Movimento do Acesso Aberto, o qual traz como desejo a superação do paradigma da comunicação científica mediada pelas grandes editoras comerciais por uma comunicação de acesso livre via internet" (RIO; LUCAS; AMORIM, 2017, p. 232)

\section{Esquecimento $n^{\circ} 02 /$ Paráfrases}

"integração"

"maior disponibilidade e rápida repercursão"

"necessidade para dispor suas produções em repositórios abertos"

"superação do paradigma da comunicação científica"

\section{Esquecimento $n^{\circ} 01 /$ Discurso}

Os A biblioteca passa daquela visão de depósito, para o principal elemento do ciclo de produção do conhecimento, disponibilizando a informação a qualquer momento e em qualquer lugar, principalmente por meio de bibliotecas digitais, ou ainda, por meio de relações semânticas entre diferentes tipos de obras - como a pintura em um museu e o filme em uma biblioteca. Os relacionamentos também são alterados, antes restritos entre o administrador e usuário, passam agora a ser multidirecionais, em que os contatos passam ser a de muitos para muitos. É ressaltado pelos autores ainda, a mudança paradigmática do conceito de usuário, antes apenas receptor da informação, agora também colabora para construção dessas informações, disponibilizando dados para a construção do conhecimento. Essas mudanças paradigmáticas também se justificam, pois as bibliotecas tiveram que acompanhar a alteração nas formas de ensino e aprendizagem, como a educação à distância, além de práticas como a disponibilização de pesquisas em repositórios institucionais auxiliarem os processos de aprendizagem em áreas economicamente desfavorecidas. Espaços como arquivos e museus também se alteram por meio do desenvolvimento de práticas de acesso aberto, visto que é recomendável que suas coleções também sejam disponibilizadas de maneira livre, conforme exposto na Declaração de Berlim.

Fonte: elaborado pelos autores (2019).

Quadro 06 - Adaptação dos processos de Organização do Conhecimento às novas tecnologias

\section{Enunciações/superfície linguística}

"As taxonomias sempre funcionaram como estruturas conceituais e de classificação para organizar e recuperar entidades de informação. Na atualidade, aplicam-se para ordenar recursos digitais alojados em servidores Web, de molde a categorizá-los e a navegar sobre eles. Converteram-se, assim, em meios de acesso, mais com uma função de visualização do que recuperação, pois ajudam os utilizadores a encontrar produtos e serviços na Web, assim como a representar melhor as suas necessidades de informação (Moreiro, 2006:97). Atuam, pois, como mapas conceituais dos assuntos sobre um campo especializado" (MEDEIROS; GONZÁLEZ, 2015, p. 03) 
“ (...) nos lleva a pensar que quizá exista una relación temática entre el objeto de estudio y la repercusión en redes sociales ya que se percibe que aquellos temas más vinculados con comunicación científica tienden a ser más difundidos en redes sociales." (FILIPPO; SILVA; BORGES, 2017, p. 43)

"Compreender o ciclo de produção académica, criando, partilhando e preservando os resultados da investigação, como referem os estudos de Haendel, Vasilevsky, e Wirz (2016) e de Koltay, Špiranec, e Karvalics (2016), exige competências de gestão e curadoria de dados decorrentes das alterações globais ao tipo e variedade de dados utilizados na investigação. A curadoria de dados deve ser, segundo estes estudos, uma competência a desenvolver não como um "penso rápido" para corresponder retrospetivamente aos mandatos nacionais para a Ciência Aberta, mas porque gerará novos insights, fazendo avançar a investigação científica. Adquirir competências para navegar numa paisagem de dados mais complexa será por si mesmo uma força motivadora para a investigação." (ANTUNES et al., 2017, p. 50)

"De um modo geral, os investigadores gerem a sua investigação e possuem competências sobre estratégias de pesquisa de informação, a avaliação dos resultados de pesquisa, a criação de alertas, a gestão de referências e a publicação de resultados. Com a Ciência Aberta são exigidas novas competências na gestão dos dados científicos, das fontes abertas e na publicação em acesso aberto. É neste entendimento que a Ciência Aberta se cruza com a literacia da informação. A Ciência Aberta é, pelo exposto, fonte e resultado da investigação científica, do ensino e da aprendizagem em contexto universitário, pelo que as instituições de ensino superior concretizam a sua missão através da Ciência Aberta. Neste contexto, as bibliotecas de ensino superior contribuem para o acesso à Ciência Aberta através da literacia da informação, ou seja, do fomento de práticas que envolvem o saber pesquisar, selecionar, avaliar e utilizar a informação. São competências que contribuem para a melhoria do desempenho dos estudantes e beneficiam o trabalho dos investigadores." (ANTUNES et al., 2017, p. 51)

"A partir da revisão de literatura, verifica-se que o serviço de referência pode ter um papel determinante na recuperação eficiente e eficaz da informação em acesso aberto já que: se foca no consumidor de informação; exige um domínio técnico e tecnológico por parte do profissional; tem um carácter profundamente adaptável; e fomenta a literacia informacional" (LEITÃO; BORGES, 2017, p. 129)

\section{Esquecimento $n^{\circ} 02 /$ Paráfrases}

"Na atualidade, aplicam-se para ordenar recursos digitais alojados em servidores Web, de molde a categorizá-los e a navegar sobre eles"

"difundidos em redes sociais"

"alterações globais ao tipo e variedade de dados utilizados na investigação"

"novos insights"

"novas competências"

Esquecimento $n^{0} 01 /$ Discurso

Verificou-se que alguns processos tradicionalmente utilizados na Organização do Conhecimento viram necessidade de adaptação ao desenvolvimento tecnológico, de forma a aperfeiçoar a recuperação documental. Com esse foco, foram empregadas linguagens como a taxonomia e a folksonomia e mapas conceituais para agregar dados na Web. Outra área da Ciência da Informação que é alterada por meio da Ciência Aberta é a construção de indicadores bibliométricos, com a criação de novos indicadores baseados em redes sociais e páginas web. A competência informacional também é influenciada nesse novo universo, visto que com um maior volume informacional, são necessárias novas competências de busca e seleção informacional, espaço que os bibliotecários de referência se tornaram ainda mais imprescindíveis.

Fonte: elaborado pelos autores (2019).

Quadro 07 - Atualização do conhecimento

Enunciações/superfície linguística

"É no âmbito do movimento do Acesso Aberto à informação e ao conhecimento, que prevê um acesso gratuito e universal que este estudo irá desenvolver-se. Entre outros produtos documentais que apresentam uma relevância substantiva nestes ambientes, salientam-se os resumos documentais, sobretudo quando estes se encontram associados às revistas científicas, pois são um dos meios mais importantes na sua divulgação e acesso." (LEITÃO; BORGES; SIMÕES, 2015, p. 02)

"En todas ellas el libre acceso a información, la colaboración científica, la transparencia y la participación de diferentes actores sociales en la construcción de conocimiento son valores centrales y el rol que se establece con el usuário (publico/ciudadano) también cambia. El concepto de "inclusión" pasa a ser protagonista." (FILIPPO; SILVA; BORGES, 2017, p. 36) 
"Este nuevo espacio de colaboración incluye un conjunto de aplicaciones propias del ámbito científico como los gestores bibliográficos sociales, e.j. Mendeley o CiteULike, las redes profesionales, e.j. ResearchGate, y las aplicaciones de identidad digital entre ellas ResearcherID u ORCID. Se completa el panorama con el movimiento de acceso abierto a la ciencia y las publicaciones y repositorios en línea (PLos ONE, ArXiv, CiteSearch, PubMed o RePEc). Esta proliferación de vías de comunicación informales constituye um nuevo reto para el análisis de la actividad científica (Mohammadi y Thelwall, 2013) y ha hecho necesario plantear nuevos modelos de abordaje y análisis de la comunicación científica" (FILIPPO; SILVA; BORGES, 2017, p. 36)

\section{Esquecimento $n^{\circ} 02 /$ Paráfrases}

"divulgação e acesso"

"construção do conhecimento como valor central"

"inclusão como protagonista"

"novos modelos de abordagem e análise da comunicação científica"

\section{Esquecimento $n^{0} 01 /$ Discurso}

Com o desenvolvimento de práticas de Ciência Aberta, os processos de desenvolvimento de conhecimento científico tornam-se mais dinâmicos, por uma questão de mais disponibilidade de dados e também porque práticas antes utilizadas visando economicidade, como quantidade de folhas ou palavras, tornaram-se dispensáveis. Outro fator que pode influenciar a atualização do conhecimento científico e que pode ser motivado pela Ciência Aberta é a colaboração científica, que pode ocorrer de maneira mais eficiente e efetiva.

Fonte: elaborado pelos autores (2019).

Quadro 08 - Transparência

Enunciações/superficie linguística

"Con la intención de alcanzar este reto europeo, la Comisión Europea presentó una propuesta de Directiva de reutilización de la información del sector público (RISP), cuya aprobación tuvo lugar a finales de 2003 con la Directiva 2003/98/CE. Dicho instrumento legal tenía como objeto la armonización del acceso a los documentos públicos en todos los organismos de los países miembros de la Unión Europea, el establecimiento de un principio de igualdad y no discriminación en el acceso y uso de la información pública o el establecimiento de un esquema transparente de precios para dicho acceso (Directiva 2003/98CE)." (COBO SERRANO; RAMOS SIMÓN; ARQUERO AVILÉS, 2015, p. 03)

"La Universidad es una de las grandes instituciones de la sociedad contemporánea y, por lo tanto, debe contemplar los valores del Gobierno Abierto (transparencia, participación y rendición de cuentas). Aunque todavía son escasos los pasos que se han dado en este sentido, este movimiento cada vez avanza más en lo que a materia de transparencia y de apertura de sus datos se refiere" (MARTÍN-GONZÁLEZ, SAINZ-IGLESIAS, 2015, p. 07) "En concreto, las políticas de transparencia responden a la necesidad de rendir cuentas ante la ciudadanía para lo que se requiere, por un lado, el cumplimiento del Principio de publicidad activa y, por otro, la regulación del derecho de acceso a la información de las instituciones sufragadas con fondos públicos entre las que se encuentran las universidades" (MARTÍN-GONZÁLEZ, SAINZ-IGLESIAS, 2015, p. 06)

"En este sentido, en los últimos años, el movimiento Open Science ha tenido un impacto notable dando lugar a diferentes iniciativas como: Open Access; Open data; investigación participativa y la ciencia ciudadana (STEPS, 2016). En todas ellas el libre acceso a información, la colaboración científica, la transparencia y la participación de diferentes actores sociales en la construcción de conocimiento son valores centrales y el rol que se establece con el usuário (publico/ciudadano) también cambia. El concepto de "inclusión" pasa a ser protagonista." (FILIPPO; SILVA; BORGES, 2017, p. 36)

"Garantizar el acceso a los resultados de investigación científica puede hacer que el sistema de investigación sea más eficiente al reducir los costos en la recolección, transferencia y reutilización de datos, al aumentar la transparencia y la calidad en el proceso de investigación, acelerar la transferência del conocimiento, aumentar la repercusión del conocimiento en la economía y promover la conexión entre los ciudadanos y la ciência" (FILIPPO; SILVA; BORGES, 2017, p. 42) 
"esquema transparente de precios para dicho acceso"

"valores del Gobierno Abierto (transparencia, participación y rendición de cuentas)."

"políticas de transparencia responden a la necesidad de rendir cuentas ante la ciudadanía"

"valores centrais"

"aumentar la transparência"

\section{Esquecimento $n^{0} 01 /$ Discurso}

Tornar acessível os dados científicos, de maneira a serem utilizados em pesquisas futuras, traz um elevado nível de transparência e garantia de que esses dados não foram manipulados e os resultados são consistentes metodologicamente. Essa transparência também é refletida em dados governamentais disponibilizados em acesso aberto, estimulando a análise e crítica de como o dinheiro público é utilizado.

Fonte: elaborado pelos autores (2019).

\section{Conclusões}

O acesso aberto trouxe significativas mudanças na divulgação de investigações científicas, dados governamentais e pesquisas acadêmicas. No entanto, pode-se perceber que o principal fator que rege todo o movimento é o acesso aberto de maneira livre, igualitária e garantindo direitos constitucionais, evocando assim o aspecto ético que essa premissa envolve.

A hipótese de pesquisa pôde ser comprovada, visto que foi possível identificar uma dimensão moral que envolve os aspectos da Ciência Aberta, como pressupostos como a transparência dos dados, retorno dos financiamentos governamentais por meio do acesso às pesquisas desenvolvidas e pagas com recursos públicos, uma forma de ir contra preços abusivos impostos pelas editoras comerciais e que prejudicavam espaços menos favorecidos financeiramente como as bibliotecas públicas, dentre outros expostos pela pesquisa.

Uma forte tendência ética também foi percebida por meio de aspectos como o acesso igualitário ao conhecimento científico, destacado pelos autores analisados. Esse teor social e democrático da Ciência Aberta está fortemente relacionado com a perspectiva moral identificada nos valores éticos. Por meio desse caminho, pode ser possível diminuir diferenças substanciais na ciência realizada em países subdesenvolvidos e de idiomas que não o inglês.

Iniciativas como os repositórios institucionais de pesquisa, além de garantir a divulgação das pesquisas produzidas, pode ir além, sendo o responsável pela criação de uma memória institucional das universidades, preservando o conhecimento socializado desses espaços. Ademais, essa forma de divulgação pode ser responsável por realizar um marketing científico da instituição, uma forma de afirmar que a universidade efetivamente realiza ciência.

Outro fator que merece destaque é a mudança paradigmática das unidades de informação que, até então, eram compreendidas apenas como espaços estáticos ou depósitos, hoje podem ser encontrados em qualquer espaço a qualquer momento. Por meio do desenvolvimento tecnológico e da perspectiva do acesso aberto, hoje os profissionais da informação participam de maneira mais interativa no desenvolvimento científico.

Embora a pesquisa tenha se centrado em um único tipo de publicação oriundo de um evento predominantemente latino-americano, percebe-se que foi possível averiguar um panorama do desenvolvimento dessa temática, que ao passar dos anos, torna-se cada vez mais evidente e atual. Sugere-se que, em futuras pesquisas, esses aspectos éticos sejam considerados, para que a Ciência Aberta se fortaleça não somente enquanto uma progressão da disponibilização do conhecimento científico, mas também uma forma de avanço social e cultural.

Agradecimentos: a autora Isadora Victorino Evangelista agradece o financiamento por meio do processo $\mathrm{n}^{\circ}$ 2017/02327-8, Fundação de Amparo à Pesquisa do Estado de São Paulo (FAPESP). 
Uma análise do discurso sobre a temática acesso aberto nos anais do EDICIC Ibérico no período de 2013 a 2017

\section{Referências}

AGUIRRE-LIGUERA, Natalia; CERETTA, Maria Gladys. Construyendo um modelo de repositório de acceso abierto para Uruguay. In: BRAVO, Blanca Rodriguez; RIBEIRO, Fernanda (Org.). Globalização, ciência e informação: atas. Porto: CETAC.MEDIA, p. 930-941, 2013. (Atas do VI Encontro Ibérico EDICIC, v. 11).

ALBAGLI, Sarita; CLINIO, Anne; RAYCHTOCK, Sabryna. Ciência aberta: correntes interpretativas de ação. Liinc em Revista, v. 10, n. 02, 2014.

ALVES, Fernanda Maria Melo; ALVES, Renato Marques. Curadoria digital: situação e perspectivas no Brasil. In: BORGES, Maria Manuel; SANZ-CASADO, Elias (Coord.). A Ciência Aberta: o contributo da Ciência da Informação. Coimbra: Universidade de Coimbra, p. 70-79, 2017. (Atas do VIII Encontro Ibérico EDICIC, v. 13).

ANTUNES, Maria da Luz et al. Literacia da informação: o primeiro degrau para a ciência aberta. In: BORGES, Maria Manuel; SANZ-CASADO, Elias (Coord.). A Ciência Aberta: o contributo da Ciência da Informação. Coimbra: Universidade de Coimbra, p. 47-56, 2017. (Atas do VIII Encontro Ibérico EDICIC, v. 13).

BAIR, S. C. Toward a code of ethics. Technical Services Quarterly, New York, v. 23, n. 1, p. 13-26, 2005.

BARROS, Thiago Henrique Bragato. Discurso, documento e arquivística: trajetória de uma área. Pesquisa Brasileira em Ciência da Informação e Biblioteconomia, v. 12, p. 97-110, 2017.

BARROS, Thiago Henrique Bragato. Uma trajetória da arquivística a partir da análise do discurso: inflexões histórico-conceituais. São Paulo: Ed da Unesp, 2016. 267p.

BUNGE, Charles A. Ethics and the reference librarian. In: LANCASTER, F. W (Ed.). Ethics and the librarian. Illinois: University of Illinois, 1991.

COBO-SERRANO, Silvia; RAMOS-SIMÓN, Luis Fernando; ARQUERO-AVILÉS, Rosario. La normativa RISP em los websites de la Administración General del Estado. In: ZABALA-VÁSQUEZ, Jon; SÁNCHEZ-JIMÉNEZ, Rodrigo; GARCÍA-MORENO, María Antonia (Coord.). Desafíos y oportunidades de las Ciencias de la Información y la Documentación en la era digital: actas del VII Encuentro Ibérico EDICIC 2015. Madrid: Universidad Complutense de Madrid, 2015. (Atas do VII Encontro Ibérico EDICIC, v. 12).

COSTA, Michelli; LEITE, Fernando César Lima. Princípios e recomendações basilares para a comunicação dos dados de pesquisa. Em questão, v. 23, n. 01, 2017.

COSTA; Teresa; ALVIM, Luísa. Uma análise bibliométrica das publicações sobre acesso aberto indexadas na Web of Science. In: BORGES, Maria Manuel; SANZ-CASADO, Elias (Coord.). A Ciência Aberta: o contributo da Ciência da Informação. Coimbra: Universidade de Coimbra, p. 113-124, 2017. (Atas do VIII Encontro Ibérico EDICIC, v. 13).

FREITAS, Maria Cristina Vieira. Revistas especializadas em termas arquivísticos de acesso aberto. In: BORGES, Maria Manuel; SANZ-CASADO, Elias (Coord.). A Ciência Aberta: o contributo da Ciência da Informação. Coimbra: Universidade de Coimbra, p. 211-226, 2017. (Atas do VIII Encontro Ibérico EDICIC, v. 13).

GUERRA-GONZÁLEZ, Jenny Teresita. Recursos ditales docentes em acceso abierto em el portal Toda la UNAM en Línea: un análisis de su visibilidad. In: ZABALA-VÁSQUEZ, Jon; SÁNCHEZ-JIMÉNEZ, Rodrigo; GARCÍA-MO RENO, María Antonia (Coord.). Desafíos y oportunidades de las Ciencias de la Información y la Documentación en la era digital: actas del VII Encuentro Ibérico EDICIC 2015. Madrid: Universidad Complutense de Madrid, 2015. (Atas do VII Encontro Ibérico EDICIC, v. 12).

GUIMARÃES, José Augusto Chaves et al. Ethics in the knowledge organization environment: an overview of values and problems in the LIS literature. In: ARSENAULT, C.; TENNIS, J. T. (Ed.). Cultural and Identity in Knowledge Organization. Würzburg: ERGON Verlag, 2008. p. 340-346. 
GUIMARÃES, José Augusto Chaves; MILANI, Suellen de Oliveira; EVANGELISTA, Isadora Victorino. Valores éticos na organização e representação do conhecimento. Encontros Bibli, Florianópolis, v. 20, n. 1, p. 19-32, 2015.

LEITÃO, Helena; BORGES, Maria Manuel; SIMÕES, Graça Melo. Resumos científicos em saúde: estudo exploratório de resumos de artigos da Nature PLoS One. In: ZABALA-VÁSQUEZ, Jon; SÁNCHEZ-JIMÉNEZ, Rodrigo; GARCÍA-MORENO, María Antonia (Coord.). Desafíos y oportunidades de las Ciencias de la Información y la Documentación en la era digital: actas del VII Encuentro Ibérico EDICIC 2015. Madrid: Universidad Complutense de Madrid, 2015. (Atas do VII Encontro Ibérico EDICIC, v. 12).

MACEDO, L. S. Ascensão. O caso dos fundos conventuais madeirenses dispersos entre o arquivo nacional torre do tombo e o arquivo regional e biblioteca pública da Madeira. In: BORGES, Maria Manuel; SANZ-CASADO, Elias (Coord.). A Ciência Aberta: o contributo da Ciência da Informação. Coimbra: Universidade de Coimbra, p. 13261344, 2017. (Atas do VIII Encontro Ibérico EDICIC, v. 13).

MACIEL; Raquel Santos; ZAFALON, Zaira Regina. Critérios para a escolha de padrões de metadados e de softwares para repositórios institucionais. In: BORGES, Maria Manuel; SANZ-CASADO, Elias (Coord.). A Ciência Aberta: o contributo da Ciência da Informação. Coimbra: Universidade de Coimbra, p. 1357-1366, 2017. (Atas do VIII Encontro Ibérico EDICIC, v. 13).

MARCONDES, Carlos H. O papel dos modelos conceituais para interoperabilidade entre acervos digitais de arquivos, bibliotecas e museus. In: ZABALA-VÁSQUEZ, Jon; SÁNCHEZ-JIMÉNEZ, Rodrigo; GARCÍA-MORENO, María Antonia (Coord.). Desafíos y oportunidades de las Ciencias de la Información y la Documentación en la era digital: actas del VII Encuentro Ibérico EDICIC 2015. Madrid: Universidad Complutense de Madrid, 2015. (Atas do VII Encontro Ibérico EDICIC, v. 12).

MARTÍN-GONZÁLEZ; Yolanda; SAINZ-IGLESIAS, Paula. Open University data em España: de los portales de transparência a los catálogos de datos abiertos. In: ZABALA-VÁSQUEZ, Jon; SÁNCHEZ-JIMÉNEZ, Rodrigo; GARCÍA-MORENO, María Antonia (Coord.). Desafíos y oportunidades de las Ciencias de la Información y la Documentación en la era digital: actas del VII Encuentro Ibérico EDICIC 2015. Madrid: Universidad Complutense de Madrid, 2015. (Atas do VII Encontro Ibérico EDICIC, v. 12).

MEDEIROS, Filipa; GONZÁLEZ, José Antonio Moreira. Profissionais da informação e medievalistas em torno da construção de uma taxonomia de história medieval portuguesa: dificuldade e reptos. In: ZABALA-VÁSQUEZ, Jon; SÁNCHEZ-JIMÉNEZ, Rodrigo; GARCÍA-MORENO, María Antonia (Coord.). Desafíos y oportunidades de las Ciencias de la Información y la Documentación en la era digital: actas del VII Encuentro Ibérico EDICIC 2015. Madrid: Universidad Complutense de Madrid, 2015. (Atas do VII Encontro Ibérico EDICIC, v. 12).

MORRISON, Samuel F. The ethics of access: towards an equal slice of the pie. In: LANCASTER, F. W (Ed.). Ethics and the librarian. Illinois: University of Illinois, 1991.

OLIVEIRA, Adriana Carla Silva de; SILVA, Edilene Maria da. Ciência aberta: dimensões para um novo fazer científico. Informação e Informação, v. 21, n. 02, 2016.

OLIVEIRA, Carla; PASTORINI, Vanessa. Portais de periódicos científicos: aspectos de visibilidade e institucionadade. In: BORGES, Maria Manuel; SANZ-CASADO, Elias (Coord.). A Ciência Aberta: o contributo da Ciência da Informação. Coimbra: Universidade de Coimbra, p. 155-164, 2017. (Atas do VIII Encontro Ibérico EDICIC, v. 13).

ORLANDI, Eni P. Análise do discurso: princípios e procedimentos. Campinas: Pontes, 2007. 100 p.

PINHEIRO, Lena Vânia. Políticas públicas, ações e manifestos de ciência aberta para pesquisa brasileira. In: BORGES, Maria Manuel; SANZ-CASADO, Elias (Coord.). A Ciência Aberta: o contributo da Ciência da Informação. Coimbra: Universidade de Coimbra, p. 271-280, 2017. (Atas do VIII Encontro Ibérico EDICIC, v. 13).

QUARESMA, Helena; BORGES, Maria Manuel. Inclusão digital: a acessibilidade das páginas web dos repositórios institucionais para deficientes. In: ZABALA-VÁSQUEZ, Jon; SÁNCHEZ-JIMÉNEZ, Rodrigo; GARCÍA-MORENO, María Antonia (Coord.). Desafíos y oportunidades de las Ciencias de la Información y la Documentación en la era 
digital: actas del VII Encuentro Ibérico EDICIC 2015. Madrid: Universidad Complutense de Madrid, 2015. (Atas do VII Encontro Ibérico EDICIC, v. 12).

RIOS; Fahima Pinto; LUCAS; Elaine Rosangela de Oliveira; AMORIM, Igor Soares. Manifestos do movimento de acesso aberto: uma análise de domínio. In: BORGES, Maria Manuel; SANZ-CASADO, Elias (Coord.). A Ciência Aberta: o contributo da Ciência da Informação. Coimbra: Universidade de Coimbra, p. 227-234, 2017. (Atas do VIII Encontro Ibérico EDICIC, v. 13).

SÁ; Maria Irene da Fonseca; SOUZA, Rosali Fernandez. Bibliotecas digitais: visibilidade e organização global. In: BRAVO, Blanca Rodriguez; RIBEIRO, Fernanda (Org.). Globalização, ciência e informação: atas. Porto: CETAC. MEDIA, p. 190-198, 2013. (Atas do VI Encontro Ibérico EDICIC, v. 11).

SAYÃO, Luis Fernando; SALES, Luana Farias. Algumas considerações sobre os repositórios digitais de dados de pesquisa. Informação e Informação, v. 21, n. 02, 2016.

SILVA, Andrieli Pachú; GUIMARÃES, José Augusto Chaves; TOGNOLI, Natália Bolfarini. Ethical values in archival arrangement and description: an analysis of professional codes of ethics. Knowledge Organization, Würzburg, v. 42, n. 5, p. 346-352, 2015.

SOUZA, Francisco das Chagas. Ética e deontologia: textos para profissionais atuantes em bibliotecas. Florianópolis: Editora da UFSC, 2002.

TARDENCILLA-MARENCO, Erick Manuel; GUANBE; Martins Fernando; ALVES, Fernanda Maria Melo. Acceso abierto em África: dificultades y desafios. In: ZABALA-VÁSQUEZ, Jon; SÁNCHEZ-JIMÉNEZ, Rodrigo; GARCÍA-MORENO, María Antonia (Coord.). Desafíos y oportunidades de las Ciencias de la Información y la Documentación en la era digital: actas del VII Encuentro Ibérico EDICIC 2015. Madrid: Universidad Complutense de Madrid, 2015. (Atas do VII Encontro Ibérico EDICIC, v. 12).

WALT, Martin Van der. Ethics in indexing classification. In: International Society for Knowledge Organization - Germany, 9., 2004, Duisburg. Proceedings... Duisburg: Universität Duisburg, 2004. Exposição oral.

WHITE, Herbert S. Teaching professional ethics to students of library and information Science. In: LANCASTER, F. W (Ed.). Ethics and the librarian. Illinois: University of Illinois, 1991 


\section{Dados dos autores}

Isadora Victorino Evangelista

Doutoranda em Ciência da Informação do Programa de Pós Graduação da Universidade Estadual Paulista isadora.evangelista@unesp.br

Thiago Henrique Bragato Barros

Professor assistente no Departamento de Ciência da Informação da Universidade Federal do Rio Grande do Sul -UFRGS

bragato.barros@ufrgs.br

Received: 2019-06-12

Accepted: 2021-07-31

\section{(c) $)$ EY}

This work is licensed under a Creative Commons Attribution 4.0 United States License.

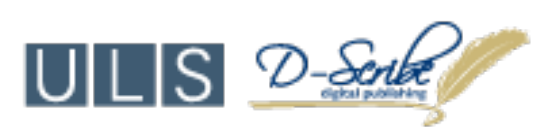

This journal is published by the University Library System of the University of Pittsburgh as part of its D-Scribe Digital Publishing Program and is cosponsored by the University of Pittsburgh Press 